\title{
Appropriate workup for lower urinary tract symptoms in men
}

\author{
Victor W. Nitti, MD \\ Professor and Vice Chairman, Department of Urology, and Director of Female Pelvic Medicine and Reconstructive Surgery, New York University Langone Medical Center, New York, NY
}

Cite as: Can Urol Assoc J 2012;6(3):S141-2. http://dx.doi.org/10.5489/cuaj.12204

\section{Abstract}

For most men with lower urinary tract symptoms (LUTS), the initial workup should consist of a careful history and physical examination, with or without simple adjunctive tests (e.g., uroflowometry, post-void residual, and frequency volume charts). Based on this basic workup, one is usually able to make an informed selection of initial treatment or watchful waiting. Urodynamics, cystoscopy and imaging are most useful in select cases when a specific reason is identified, the diagnosis is uncertain or when patients have failed simple initial treatments and seek further therapy.

ower urinary tract symptoms (LUTS) are extremely common in men and increase with age. LUTS can be broadly divided into storage symptoms (urgency, frequency, urgency incontinence, nocturia) and voiding symptoms (slow stream, hesitancy, feeling of incomplete emptying). Voiding and storage symptoms often coexist. In general, voiding symptoms are caused by either outlet obstruction or impaired contractility, while storage symptoms are related to detrusor overactivity, increased bladder sensitivity or impaired compliance. Before treating LUTS, it is important to ascertain the type of symptoms (voiding, storage, or both), as well as the severity and degree of bother of both. The amount of information needed from the workup depends upon the complexity of symptoms, presence of comorbidities (e.g., neurological disease, diabetes) and any abnormalities found in the initial basic workup (e.g., very high post-void residual [PVR]). The following review discusses what the author feels is an appropriate workup of a male patient with LUTS. In discussing implementation of initial or simple treatments we are referring to behavioural therapy, pharmacologic therapy (e.g., alpha-blockers, 5-alpha reductase inhibitors, antimuscarinics) and physiotherapy.

\section{Initial basic workup of male LUTS}

In most men, a simple workup is sufficient to initiate first-line treatments such as behavioural modification and pharmacotherapy (e.g., alpha-blockers, 5-alpha reductase inhibitors, or antimuscarinics) depending on the initial assessment.

\section{History}

A detailed medical history should focus on the urinary tract and aim at identifying conditions (other than benign and malignant disorders of the prostate) that may be responsible for the LUTS. ${ }^{1}$

Asking a patient to complete a frequency volume chart can be a very helpful corollary to the history. It is useful for all storage LUTS and is essential for nocturia when it is an isolated complaint. The parameters that should be assessed in such a chart include the total number of voids per 24 hours; the total number of daytime (awake) voids; the total number of nighttime voids; total fluid intake; total voided volume; maximum, minimum and mean voided volume; and the number of episodes of urgency and urge urinary incontinence. Ideally, the patient should be asked to complete at least three 24-hour diaries, though a single diary can still be useful.

\section{Physical examination}

The physical examination should include assessment for the presence or absence of a distended bladder, excoriation of the genitals secondary to urinary incontinence and evidence of urethral discharge. Other assessments that are considered essential in the workup of LUTS are a focused neurological examination and assessment of gait, sphincter tone, anal wick, bulbocavernosis reflex and LE reflexes. A digital rectal examination is also recommended.

\section{Validated symptom scores}

Validated symptom scores can also be very helpful in providing a more complete understanding of the patient's LUTS, helping to categorize the type, severity and degree of bother of different symptoms. The most commonly used tools are: the International Prostate Symptom Score (IPSS) for general male LUTS and for LUTS suggestive of bladder outlet obstruction (BOO); the overactive bladder questionnaire $(\mathrm{OAB}-\mathrm{q})$ for the patient exhibiting primarily storage symptoms; and the International Consultation on Incontinence Questionnaire (ICIQ) for patients with urinary incontinence. 


\section{Simple office tests}

Simple office tests such as uroflowometry and PVR determination are important tools to assess bladder emptying in a non-invasive way.

Uroflowometry is a non-invasive assessment that can provide an objective and quantitative indication of the integration of bladder and outlet function among patients with voiding symptoms. Significant abnormalities are indicative of a dysfunction in the voiding phase of micturition cycle. Uroflowometry can also be used to monitor treatment outcomes and correlate symptoms with objective findings. However, the test cannot distinguish outlet obstruction from bladder underactivity, and the test's dependence on voided volume may cause significant variability of measured uroflows in the same patient.

While there is no consensus on what constitutes an elevated or dangerous PVR volume, in some patients an elevated PVR may be harmful. Clinicians may perform a PVR in patients with LUTS as a safety measure to rule out significant urinary retention both initially and during follow-up. However, one needs to keep in mind the limitations of the information provided by this test. The PVR does not differentiate obstruction from other causes, and no relevant studies have identified the usefulness of PVR for guiding clinical management, improving or predicting outcomes of treatment.

\section{Recommended workup for more complex cases}

In more complex cases, when first-line treatments fail or the patient has a very high PVR, neurological disease or other worrisome findings, ancillary testing like urodynamics and/or cystoscopy may be useful to make a more precise diagnosis and focus treatment, especially if that second-line treatment has significant morbidity or expense. In general, if invasive testing is performed, the results of the test should have an effect on patient treatment and/or counselling.

\section{Urodynamics}

Urodynamics are indicated in the event of failure of what appears to be appropriate initial therapy, if the diagnosis is uncertain or if the next step in treatment is invasive or expensive. It may also be used to assess patients with significant incontinence (especially if this is the chief complaint), patients with very high PVR (particularly if watchful waiting is considered), those with neurologic disease and in cases where there is uncertainty in how to proceed with treatment. For men considering surgery for the treatment of LUTS secondary to benign prostatic obstruction, almost all evidence for the advantages of UDS prior to invasive therapy is Level 3 (good quality retrospective "case-control studies" or "case series"), the quantity of evidence allows a Grade B recommendation to preform UDS testing according to the International Consultation on Urologic Diseases. ${ }^{2}$

\section{Cystoscopy}

Cystoscopy should be preformed patients with associated hematuria, a history of transitional cell carcinoma, possible intravesical or urethral lesion, refractory overactive bladder prior to neuromodulation or onabotulinuntoxinA injection, or if cystoscopy would alter type of treatment selected.

\section{Imaging}

Imaging of the upper urinary tract can be helpful for patients with an elevated PVR, to rule out hydronephrosis, and in patients with LUTS associated with renal insufficiency. Transrectal ultrasound (or other prostate imaging) may be considered for cases where the results of the imaging would affect treatment selection.

\section{Conclusions}

In most cases of LUTS in men, initial treatment or watchful waiting can be initiated based on a history and physical exam. Uroflowometry, PVR, and frequency volume charts are useful adjuncts that are relatively cost effective.

Urodynamics, cystoscopy and imaging are most useful in select cases when a specific reason is identified, the diagnosis is in question or when patients have failed simple initial treatments and are considering further therapy.

Competing interests: Dr. Nitti is an ongoing paid consultant with Allergan, Astellas, Coloplast, Medtronic, Serenity Pharmaceuticals and Uroplasty.

\section{References}

1. McConnell J, Abrams P, Denis L, et al (eds). Male Lower Urinary Tract Dysfunction: Evaluation and Management. International Consultation on Urological Diseases. Health Publications, Paris; 2006.

2. Abrams $P$, Cardozo L, Fall M, et al. 6th International Consultation on New Developments in Prostate Cancer and Diseases, June 24-27, 2005, Paris, France.

Correspondence: Dr. Victor Nititi, NYU Urology Associates, 150 East 32nd St., 2nd Floor, New York, NY 10016; victor.nitti@nyumc.org 